\title{
The Viscous Properties of Diols. V. 1,2-Hexanediol in Water and Butanol Solutions
}

\author{
Paweł Jarosiewicz, Grzegorz Czechowski, and Jan Jadżyn \\ Institute of Molecular Physics, Polish Academy of Sciences, \\ M. Smoluchowskiego 17, 60-179 Poznań, Poland \\ Reprint requests to Prof. J.J.; Fax: +4861 8684-524, E-mail: jadzyn@ifmpan.poznan.pl
}

Z. Naturforsch. 59a, 559 - 562 (2004); received May 31, 2004

\begin{abstract}
The paper presents the results of viscosity measurements performed on 1,2-hexanediol in water and $n$-, $s$ - and $t$-butanol solutions in the whole range of concentrations, at different temperatures. The activation energy for viscous flow of the solutions and the viscosity excess, were determined.
\end{abstract}

Key words: Shear Viscosity; Hexanediol; Water; Butanols; Solutions; Viscosity Excess; Activation Energy.

\section{Introduction}

The viscosity of liquids plays a crucial role in many technical processes, and great effort is made in finding liquids of an appropriate viscosity with its temperature dependence as weak as possible. For thermally activated processes, as the viscous flow, a weak temperature dependence corresponds to a small activation energy of the process.

The diols, as compounds of an exceptionally large temperature range of their existence in the liquid state, are widely used in practice. All peculiarities in the physical properties of the diols are due to intermolecular hydrogen bonds formed by hydroxyl groups. The hydrogen bonds make the molecular organization in liquid diols quite stable, but the structure of the intermolecular entities depends mainly on the mutual position of $\mathrm{OH}$ groups within the hydrocarbon chain of the diol molecule. The entities formed by the diol molecules are a good example of supramolecular polymers [1-4] in which the molecules are linked by reversible, non-covalent bonds and, as a result, the degree of polymerization and the mean molecular weight can be easily regulated by changing the concentration of the diol in the solvent or by changing the temperature.

This paper presents the results of viscosity measurements performed on widely used mixtures of 1,2hexanediol and water or three isomers of butyl alcohol.

\section{Experiment}

1,2-Hexanediol (Aldrich) of 98\% purity, and $n-$, $s$-, and $t$-butanol (2-methyl-2-propanol), (Aldrich, $>99 \%$ ), were used as purchased. The water was twice distilled and deionized.

The shear viscosity was measured with a Haake rotator viscometer RV20 with an accuracy of $0.5 \%$. The temperature of the sample was controlled within $\pm 0.1{ }^{\circ} \mathrm{C}$. The experimental details are described in [5].

\section{Results and Discussion}

Figure 1a presents temperature dependences of the viscosity of 1,2-hexanediol dissolved in water. The mole fractions of the diol $(x)$ are the parameters of the curves. The viscosity of pure water is almost too small to appear in the figure. The solid lines present the best fit of the Arrhenius equation

$$
\eta(T)=\eta_{\mathrm{A}} \exp \left(\frac{E_{\mathrm{A}}}{R T}\right)+\eta_{\mathrm{o}}
$$

to the experimental data. In (1) $\eta_{\mathrm{o}}$ denotes the viscosity background [6] (in practice about $1 \mathrm{mPas}$ ).

Figure $1 \mathrm{~b}$ presents the viscosity dependence on the 1,2-hexanediol concentration in water, at constant temperature, and Fig. 1c the dependence of the viscosity excess,

$$
\eta^{E}=\eta_{\text {meas }}-\left[\eta_{\text {diol }} \cdot x+\eta_{\text {solv }} \cdot(1-x)\right]
$$



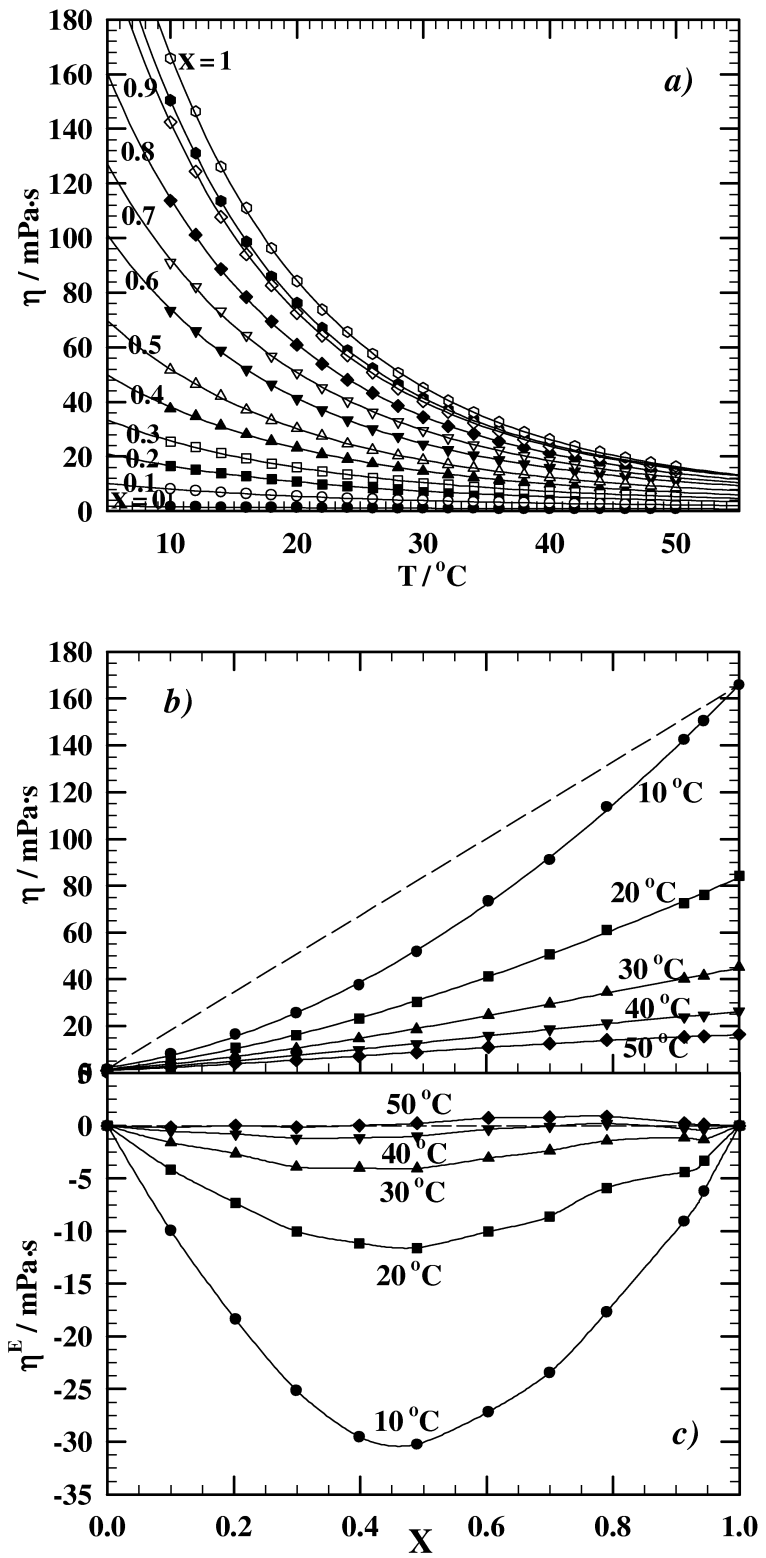

Fig. 1. The viscous properties of 1,2-hexanediol + water solutions: a) the viscosity of the solutions as a function of temperature, $(x$ denotes the mole fraction of the diol $), b)$ isothermal viscosity and c) the viscosity excess as a function of the mole fraction of the diol.

where $\eta_{\text {meas }}$ is the measured viscosity of the solution and $\eta_{\text {diol }}$ and $\eta_{\text {solv }}$ are the viscosities of the pure components of the mixture.

$\eta(T), \eta(x)$ and $\eta^{E}(x)$, obtained for the mixtures of 1,2-hexanediol with $n$-, $s$ - and $t$-butanol, are presented in Figs. 2, 3, and 4, respectively.

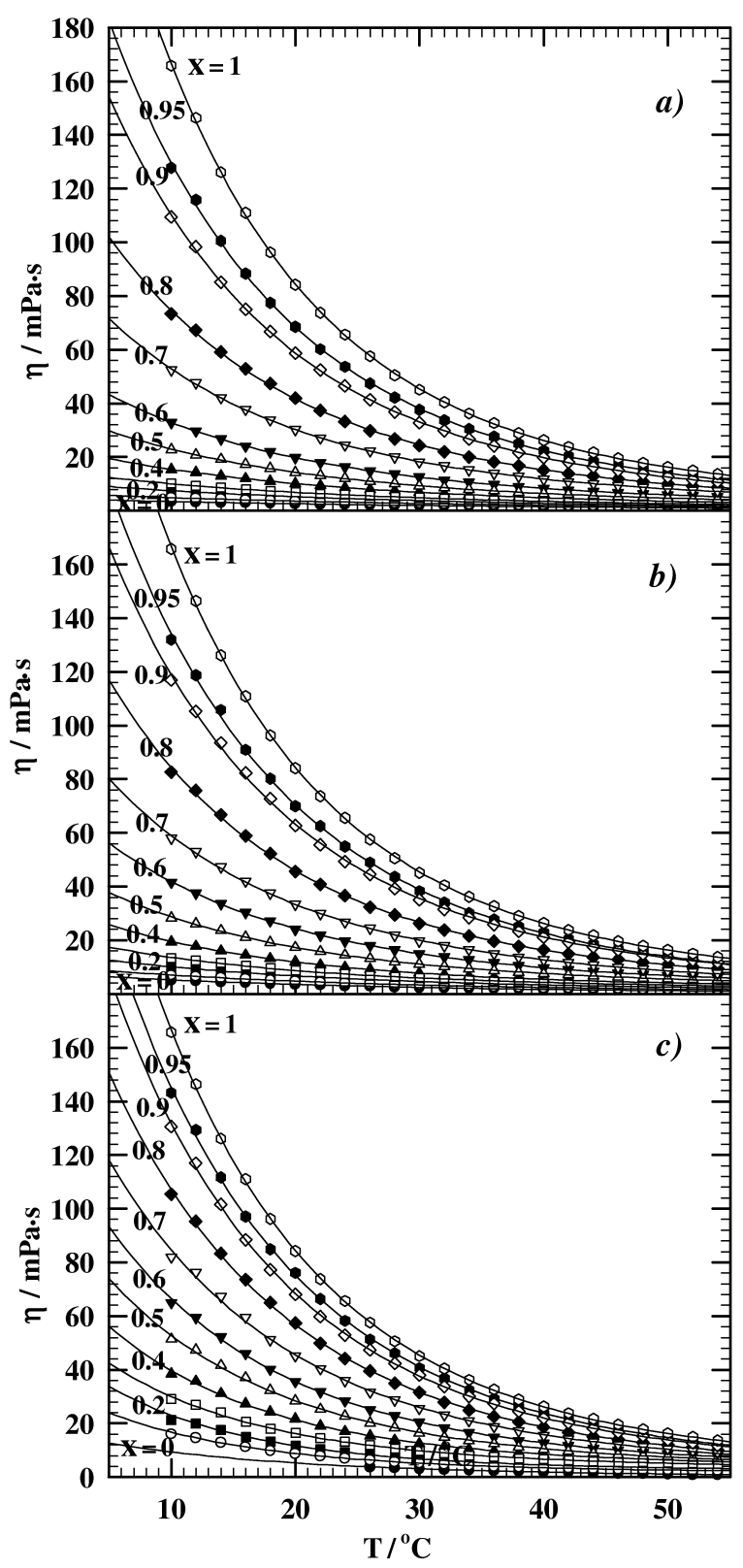

Fig. 2. Temperature dependences of the viscosity of 1,2 hexanediol in $n$-butanol (a), $s$-butanol (b) and $t$-butanol (c) solutions. The solid lines represent the best fit of (1) to the experimental data.

The first conclusion, which can be important for the practical reasons, concerns the excess of the mixtures viscosity. The dissolvation of 1,2-hexanediol both in water and the butanols leads to a decrease of the viscosity, i. e. to a negative excess of the viscosity. Some exceptions can be found at higher temperatures, where 


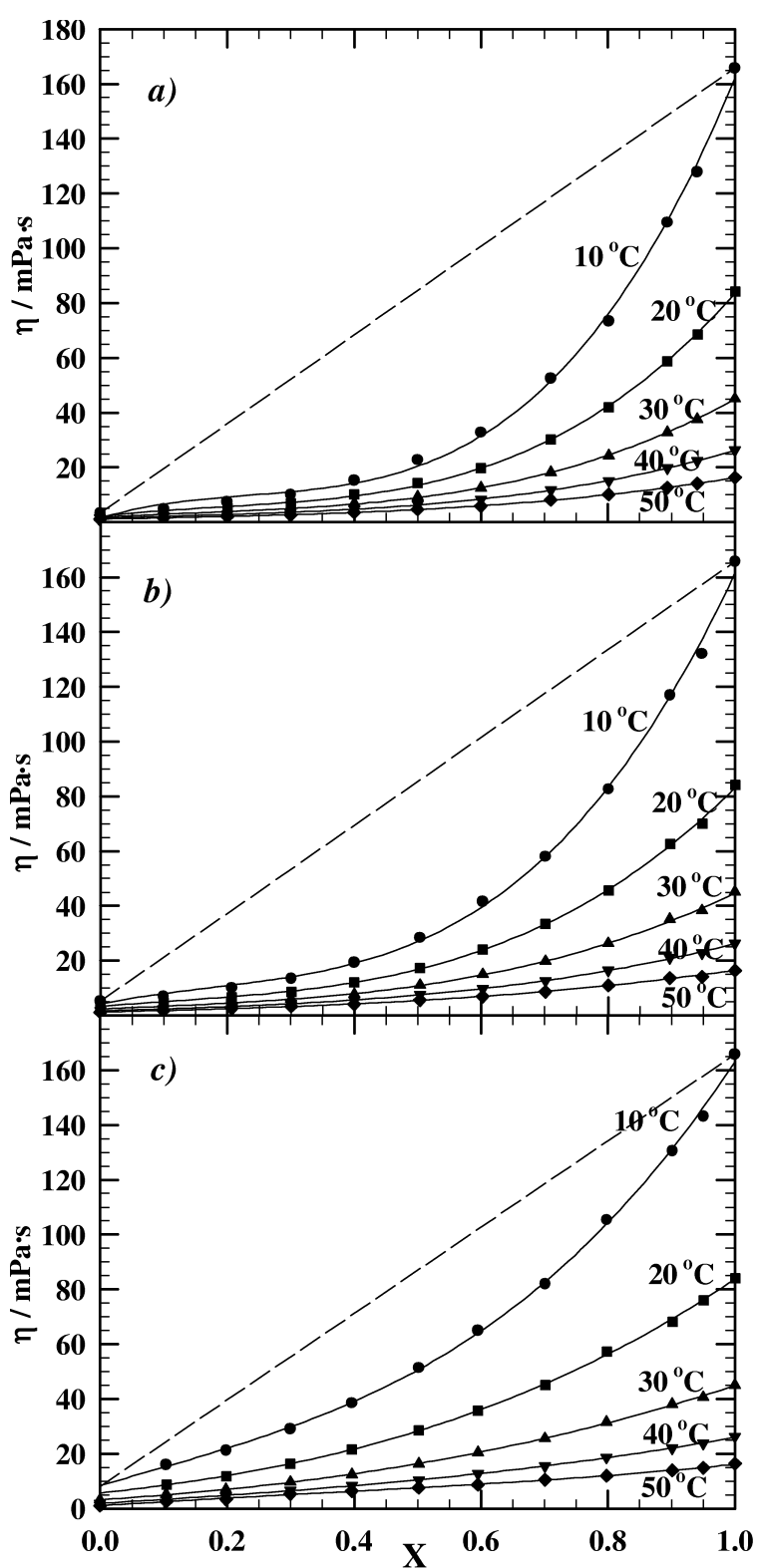

Fig. 3. Isothermal viscosity dependences on the 1,2 hexanediol mole fraction in $n$-butanol (a), $s$-butanol (b) and $t$-butanol (c) solutions.

in a limited range of the diol concentration a small positive viscosity excess appears. At $10{ }^{\circ} \mathrm{C}$, the minimum of $\eta^{E}$ gains quite important values, which show a decrease as the solvent changes in the series $n-, s-$, $t$-butanol in water. It is not a hazardous succession, because in this way the shape of the solvent molecules gradually changes from the most elongated to close to the spherical one. Such an effect of the shape of

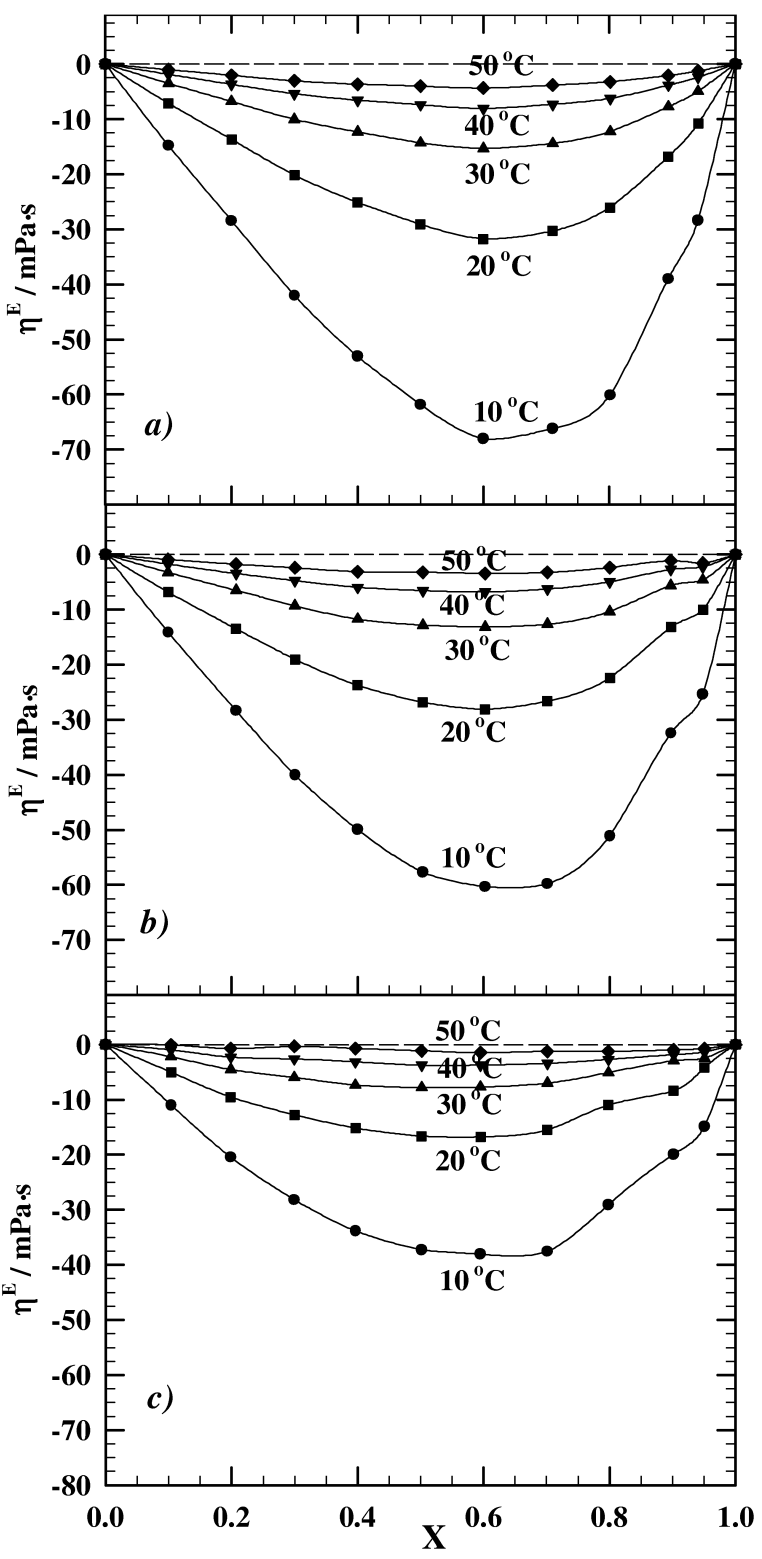

Fig. 4. Viscosity excess as a function of the mole fraction of 1,2-hexanediol in $n$-butanol (a), $s$-butanol (b) and $t$ butanol (c) solutions.

the solvent molecules is expected because, due to the separation of the hydrophobic and hydrophilic parts in 1,2-hexanediol molecules, the intermolecular entities formed have a micelle-like structure [7], which is more compatible to the spherical shaped molecules of admixtures, than to the elongated ones.

The second conclusion concerns the viscosity activation energy determined for the solutions studied. As 


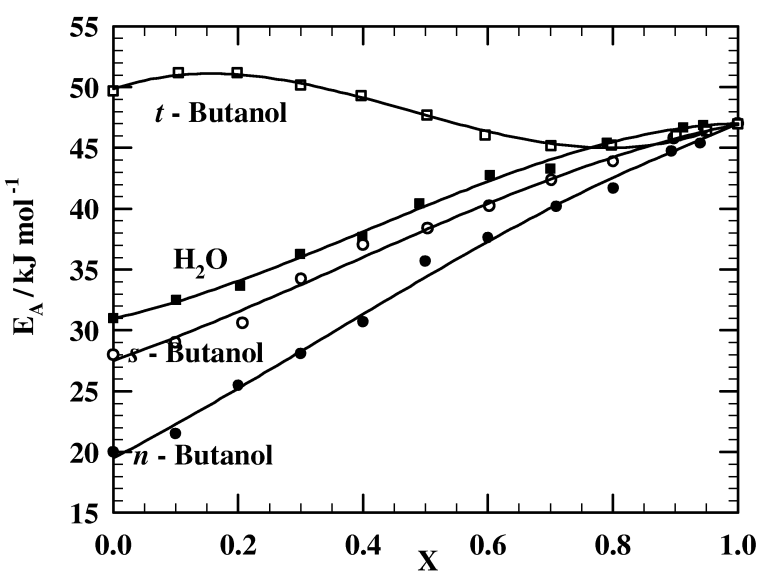

Fig. 5. Viscosity activation energy as a function of the mole fraction of 1,2-hexanediol dissolved in water and butanols.

[1] R.P. Sijbesma, F.H. Beijer, L. Brunsveld, B. J. B. Folmer, J. H. K. Ky Hirschberg, R. F. M. Lange, J. K. L. Lowe, and E. W. Meijer, Science 278, 1601 (1997).

[2] V. A. Durov, J. Molec. Liq. 103-104, 41 (2003).

[3] S. Boileau, L. Bouteiller, F. Laupretre, and F. Lortie, New J. Chem. 24, 845 (2000).

[4] J. Jadżyn, M. Stockhausen, and B. Żywucki, J. Phys. Chem. 91, 754 (1987). seen in Fig. 5, as the concentration of 1,2-hexanediol increases, the activation energy changes monotonically from the value corresponding to the pure butanols or water, to that corresponding to the pure diol. It means that one cannot expect to find some mixture of 1,2hexanediol + butanols or water for which the viscosity temperature dependence will be reduced in comparison to the pure component of the mixture - the effect would be advantageous from the technical point of view.
[5] G. Czechowski and J. Jadżyn, Z. Naturforsch. 58a, 317 (2003).

[6] J. Jadżyn, G. Czechowski, and T. Lech, Acta Phys. Polon. 101, 495 (2002).

[7] A. G. Petrov, The Lyotropic State of Matter, Gordon and Breach Sci. Publ., Amsterdam 1999. 